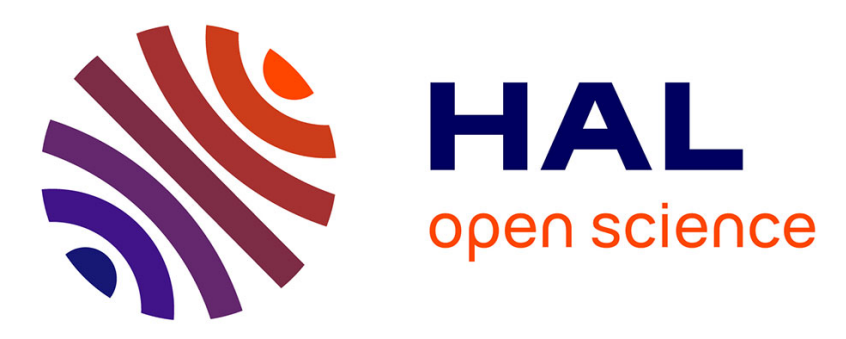

\title{
Fairness-Energy-Throughput Optimized Trade-off in Wireless Networks
}

Cédric Gueguen, Malo Manini

\section{To cite this version:}

Cédric Gueguen, Malo Manini. Fairness-Energy-Throughput Optimized Trade-off in Wireless Networks. ICNC 2018 - International Conference on Computing, Networking and Communications, Mar 2018, Maui, United States. pp.1-8. hal-01688870

\section{HAL Id: hal-01688870 https://hal.science/hal-01688870}

Submitted on 22 Jan 2018

HAL is a multi-disciplinary open access archive for the deposit and dissemination of scientific research documents, whether they are published or not. The documents may come from teaching and research institutions in France or abroad, or from public or private research centers.
L'archive ouverte pluridisciplinaire HAL, est destinée au dépôt et à la diffusion de documents scientifiques de niveau recherche, publiés ou non, émanant des établissements d'enseignement et de recherche français ou étrangers, des laboratoires publics ou privés. 


\title{
Fairness-Energy-Throughput Optimized Trade-off in Wireless Networks
}

\author{
Cédric Gueguen, Malo Manini \\ B-com, University of Rennes1/IRISA \\ France \\ Email: \{cedric.gueguen, malo.manini\}@B-com.com; \{cedric.gueguen\}@irisa.fr;
}

\begin{abstract}
Increase the spectral efficiency and guarantee high Quality of Service (QoS) and high Quality of Experience (QoE) is the crucial issue of wireless communications. The best acknowledged opportunistic resource allocation schedulers allow to reach these objectives taking into consideration the radio conditions and users requirement in the allocation process. However, this is often process at the expense of energy efficiency which is now essential regarding the alarming greenhouse gas emission and the need to improve the device battery lifetime. This paper proposes an optimized trade-off between energy, throughput and fairness thanks to a new opportunistic approach that combines the advantages without the drawbacks of specialized schedulers. Performance evaluations show that the proposed solution allows to have the same system capacity that MaxSNR scheduler while solving its lack of fairness concerning mobiles at different distances from the access point. In addition we also show that this can be made lowering energy consumption.

Index Terms-Opportunistic Scheduling, Quality of Service,
\end{abstract} Fairness, Energy Consumption, Multiuser diversity.

\section{INTRODUCTION}

The increasing number of users which each requires more and more throughput and tight QoS requirement drive us to develop new resource allocation algorithms that optimize global system throughput while ensuring high fairness. In addition, guarantee high QoE cannot be reach without offering a good and substainable mobility. This require that the new resource allocation strategies provide high energy consumption efficiency in order to increase battery lifetime.

The resource allocation strategies traditionally used in wireless networks were originally and primarily designed for the wired context. These conventional access methods like Round Robin (RR) and Random Access (RA) are not well adapted to the wireless environment and provide very poor throughput. Intensive research efforts have been given in order to propose throughput efficient schedulers and opportunistic approaches have emerged as the best way. The best known is called Maximum Signal to Noise Ratio (MaxSNR) scheduler [1], [2]. It preferably allocates the resources to the active user(s) with the most favourable channel conditions at a given time. It takes benefit of multiuser and frequency diversity in order to maximize the system throughput (Fig. 1). However users close to the access point have a better average throughtput per Resource Unit (RU) than far users. This induce that, with MaxSNR scheduler, close users have statistically more chances to have access to the medium. In consequence, far users will often obtain radio resources after close users making them overpassing their QoS requirement.

To answer this fairness issue several solutions have beed proposed. The PF is the best acknowledged [3], [4], [5]. It optimizes the system capacity while ensuring a good fairness. However, we recently propose some solutions that outperform PF considering system capacity as well as fairness [6], [7]:

- Weighted Fair Opportunistic Scheduler (WFO) [6] applies cross-layer design concepts taking into account both the physical layer specificities (transmission conditions) and the higher layer constraints (traffic patterns, QoS constraints). Physical layer informations are used in order to take advantage of the time, frequency and multiuser diversity and maximize the system capacity. Higher layer informations are exploited in a weighted system that introduces dynamic priorities between flows for ensuring the same QoS level to all mobiles. This results in a scheme which guarantees the differentiated QoS constraints (data integrity and delay targets) of heterogeneous traffic flows. However, even if highly efficient and widely outperforming PF, WFO need to compute and signal additionnal parameters that make it hard to implement in current networks.

- Fair MaxSNR (FmaxSNR) [7] provides the same benefit than WFO concerning the fairness issue between mobile located at different distance of the access point but staying very simple to implement. Its principle is to add, in the MaxSNR algorithm, a correction factor based on the mean Signal to Noise Ratio (SNR) value. This allows to compensate the path loss and shadowing negative effect on MaxSNR fairness. In addition, since all opportunistic schemes strongly rely on mutliuser diversity for increase global system throughput, FMaxSNR improves the MaxSNR system capacity optimization properties. Indeed, MaxSNR maximize the throughput at short time scale but, generally serving close user before the other, it does not take benefit of all the opportunities and this does not provide the best system capacity at long time scale. On the contrary, FmaxSNR finely and simultaneously manages all mobiles together. Thanks to its fair approach, it preserves the multiuser diversity and takes a maximal benefit of the opportunistic approaches. This results in a better spectral efficiency than MaxSNR and PF.

All these schemes are fair and offer good system capacity properties, however they are very greedy in energy. In order to offer more battery autonomy to users, other solution focusing on energy have been developt. The Power-based proportional fairness (PPF) [8] proposes to reduce energy consumption avoiding the allocation with low SNR and delaying flows that have worst channel condition. This provides good energy 


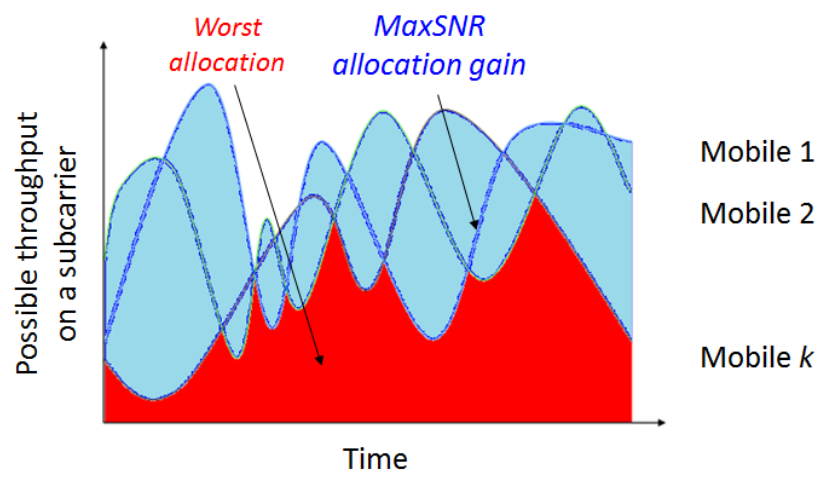

Fig. 1. MaxSNR opportunistic system capacity increase.

efficiency since this gives access to the medium only to users with good SNR and allows to always use highest modulation that are the most profitable. However, the best way to minimize energy consumption is not only to optimize the modulation but mainly to maximize the sleep time. The Opportunistic Energy Aware scheduler (OEA) [9] is built on this principle. It exploits active-sleep mode and channel condition together. While other schedulers can potentially active all users, the OEA limits this number. This allows to compress the transmission time (i.e. active mode), greedy in energy. Considering the channel condition in the allocation process, only allocations with good modulation are also conserved. However these both schedulers lack of fairness. Users far from the access point have statistically worst SNR than close users and like with MaxSNR will generally be served in a second step. Since energy efficiency guarantee must not evade QoS requirement and the system capacity optimization, new approaches must be developt in order to bring together: high spectral efficiency, fairness and energy consumption minimization.

In this paper we propose an opportunistic scheduler that guarantee a Fairness-Energy-Throughput Optimized Tradeoff. This solution called FETOT takes into account of the radio condition in order to avoid bad allocation in term of throughput. The FmaxSNR correction factor on the distance is adequalty integrated in the algorithm in order to offer the same high fairness considering far and close users. This scheduler is also built to compress the transmission time but, contrary to the OEA, FETOT is able to take a full benefit on the multiuser diversity thanks to a new trade-off parameter. The result is that FETOT combines the advantages of MaxSNR, FMAXSNR and OEA respectivly on system capacity, fairness and energy efficiency.

This paper is organized as follows. Second section proposes a description of the system under study. Third section describes our FETOT proposition. Fourth section presents a detailed performance evaluation and last section concludes the paper.

\section{CONTEXT DESCRIPTION}

In this paper, we focus on the allocation of radio resources among the set of users situated in the coverage zone of an access point. We consider a centralized and synchronized approach compatible with opportunistic resource allocation techniques [10]. Our work is designed for Multiple Input Multiple Output (MIMO) - OFDM technology which is acknowledged for outperforming other strategies in terms of spectral efficiency [11]. The total available bandwidth is divided in sub-frequency bands, i.e. subcarriers. The radio resource is further divided in the time domain in frames. Each frame is itself divided in Time Slots (TS) of constant duration. The TS duration is an integer multiple of the OFDM symbol duration. The number of subcarriers is chosen so that the width of each sub-frequency band is less than the coherence bandwidth of the channel. Moreover, the frame duration is fixed to a value much smaller than the coherence time of the channel. With these assumptions, the transmission on each subcarrier is subject to flat fading with a channel state that can be considered static during each frame. However, the transmissions performed on different subcarriers by different users are assumed to have independent channel state values [12]. In addition, the elementary resource unit (RU) is defined as any (subcarrier, TS) pair. Each of this RU may be allocated to any user with a specific modulation order. On each RU, the modulation scheme is QAM with a modulation order adapted to the channel state between the access point and the user to which it is allocated. This provides the flexible resource allocation framework required for opportunistic scheduling.

\section{Opportunistic Energy Aware Scheduler}

The FETOT scheduling algorithm relies on weights that set the dynamic priorities for allocating the radio resources. These weights are built in order to satisfy three major objectives: system capacity maximization, fairness and energy consumption minimization as explained below.

\section{A. System Throughput Maximization}

The FETOT scheduler optimizes the system throughput in a MAC/PHY opportunistic approach. At each frame allocation, the scheduler computes the maximum number of bits $q_{k, n}$ that can be transmitted in a TS of subcarrier $n$ if assigned to user $k$ while keeping below its BER target $\left(B E R_{\text {target }, k}\right)$, for all $k$ and all $n$ :

$$
q_{k, n} \leq\left\lfloor\log _{2}\left(1+\frac{3 P \times T_{s} \times\left(\frac{1}{d_{k}}\right)^{3.5} \times \alpha_{k, n}^{2}}{2 N_{0}\left[e r f c^{-1}\left(\frac{B E R_{\text {target }, k}}{2}\right)\right]^{2}}\right)\right\rfloor
$$

where $P$ is the transmission power, $N_{0}$ is the spectral density of noise, $T_{s}$ is the OFDM symbol duration, $d_{k}$ is the distance to the access point of the user $k$ and $\alpha_{k, n}^{2}$ represents the flat fading experienced by this user on subcarrier $n$. In the following, $\alpha_{k, n}$ is Rayleigh distributed with an expectation equal to unity. Due to multi-path fading, the potential number of bit that a user can transmit on a RU will fluctuate around this value over the time.

We further assume that the supported QAM modulation orders are limited such as $q$ belongs to the set $S=$ $\left\{0,2,4, \ldots, q_{\max }\right\}$. Hence, the maximum number of bits $m_{k, n}$ that will be transmitted on a TS of subcarrier $n$ if this RU is allocated to the user $k$ is: 


$$
m_{k, n}=\max \left\{q \in S, q \leq q_{k, n}\right\} .
$$

MaxSNR based schemes allocate the RU to the user which have the greatest $m_{k, n}$ values. This strategy maximizes the system capacity at short time scale but is highly unfair considering users far to the access point that are often delaying out of their delay requirement. In order to provide more fairness considering users locations while preserving the system throughput maximization, a fairness parameter is introduced in FETOT.

\section{B. Fairness guarantee}

FETOT integrates in its scheduling process the fairness parameter proposed in [7]. Called "Compensation Factor" $\left(C F_{k}\right)$, this parameter takes into account the current path loss impact on the average achievable bit rate of mobile $k$ :

$$
C F_{k}=\frac{b_{r e f}}{b_{k}} \text {. }
$$

$b_{r e f}$ is a reference number of bits that may be transmitted on a subcarrier considering a reference free space path loss $a_{r e f}$ for a reference distance $d_{r e f}$ to the access point and a multipath fading equal to unity:

$$
b_{\text {ref }}=\log _{2}\left(1+\frac{3 P_{\max } \times T_{s} \times a_{\text {ref }}}{2 N_{0}\left[e r f c^{-1}\left(\frac{B E R_{\text {target }}}{2}\right)\right]^{2}}\right) .
$$

$b_{k}$ represents the same quantity but considering a distance $d_{k}$ to the access point:

$$
b_{k}=\log _{2}\left(1+\frac{3 P_{\max } \times T_{s} \times a_{\text {ref }} \times\left(\frac{d_{\text {ref }}}{d_{k}}\right)^{\beta}}{2 N_{0}\left[e r f c^{-1}\left(\frac{B E R_{\text {target }}}{2}\right)\right]^{2}}\right),
$$

with $\beta$ the experienced path loss exponent.

Adequalty combining and taking into account both $m_{k, n}$ and $C F_{k}$ in the allocation process, FETOT considers all mobiles virtually at the same position in the scheduling decision. $C F_{k}$ adequately compensates the lower spectral efficiencies of far mobiles bringing high fairness in the allocation process. An equal throughput can be provided to each mobile while keeping the MaxSNR opportunistic scheduling advantages thanks to the $m_{k, n}$ parameters which take into account the channel state. Moreover, in contrast with MaxSNR and PF which satisfy much faster the mobiles which are close to the access point, FETOT keeps more mobiles active but with a relatively low traffic backlog. Satisfaction of delay constraints is more uniform and, better preserving the multiuser diversity, a more efficient usage of the bandwidth is made. This jointly ensures fairness and system throughput maximization like FMaxSNR. If two mobiles have an equal priority for and $\mathrm{RU}$, this one is given to the mobile which has the highest buffer occupancy further strengthening fairness. At this step, FETOT optimizes the throughput and guarantee high fairness but highly suffers of a inefficient energy management. In order to provide energy consumption minimization while preserving the system throughput maximization and fairness, an energy parameter is introduced.

\section{Energy consumption minimization}

The third major objective of the FETOT is to provide efficient energy management in addition to the system throughput optimization. Existing opportunistic resource mapping (as MaxSNR, PF or FMaxSNR for example) are basically horizontals . Due to flat fading during a frame, often a same user strictly experienced the greatest channel condition on each TS of a subcarrier. Consequently, with classical opportunistic schedulers, a same user often receives all the TS of a subcarrier and need to stay in active mode during a long time. Note that we can potentially have one different selected user on each available subcarrier. Consequently, during all TS, many selected users can not be set in sleep mode. They consume a lot of power to transmit few bit during a long time (with many allocated TS but on few subcarriers). To conclude, opportunistic scheduling is acknowledged as the best way to manage wireless resources, maximizing the system capacity and providing QoS but they need to be more energy efficient.

The FETOT scheduler integrates a modified version of OEA solution keeping these energy benefit without their fairness and system capacity failure. We propose to drastically minimize the energy consumption in particular by increasing the sleeping mode duration. In order to achieve this goal, FETOT extends the classical opportunistic cross-layer design to obtain a new vertical opportunistic resource mapping. When a user is in active mode, FETOT tries, like OEA, to benefit from its activation in order to compress its time of activity and to transmit more bit per "used" TS. Like this, FETOT allows to significantly increase sleeping mode duration and energy preservation. Originally, OEA scheduler computed an "Energy Transmission Cost" $\left(E T C_{k}\right)$ parameter (in Watt). It is based on the energy cost of user $k$ to transmit on a RU:

$$
E T C_{k}=A_{k} * C n_{k}+\left(1-A_{k}\right) *\left(C_{k}+C n_{k}\right),
$$

When the user $k$ is in active mode, $A_{k}=1$ else, $A_{k}=0$ (i.e. sleep mode). In addition, $C n_{k}$ and $C_{k}$ are two constants (in Watt). $C_{k}$ represents the energy needed to wake up the user $k$ from the sleep mode to the active mode. $C n_{k}$ represents the energy needed to transmit on a $n^{t h}$ allocated subcarrier. Note that $C_{k}>C n_{k}$ since the energy needed to be active is highly superior to the amount of energy required to transmit on an additionnal RU if user already set in active mode.

$E T C_{k}$ is used in OEA scheduler but have the negative side effect to highly reduce the usage done of the multiuser diversity. This drastically and negatively impact the OEA system capacity optimization. In order to keep its energy minimization properties while fixing this throughput issue, FETOT integrate a modified $E T C_{k}$ parameter that we called “Throughput-Energy Tradeoff" parameter $T E T_{k}$ :

$$
T E T_{k}=A_{k} * C n_{k}+\left(1-A_{k}\right) *\left(\frac{C_{k}}{M D F}+C n_{k}\right),
$$

where $M D F$ is a Multiuser Diversity Factor. The higher $M D F$ is, the more the system will try to increase the number of active user at the same time, increasing the multiuser usage and consequently the global system throughput at the expense of the energy consumption (infinite $M D F$ value makes $T E T_{k}$ 
constant and induces FETOT similar to a FMaxSNR resource allocation). At the opposite, low $M D F$ value make FETOT deacreasing the number of active user at the same time, reducing energy consumption at the expense of the multiuser diversity usage that provides a resource allocation close to OEA scheduling (excepting that this version of the OEA should be strongly more fair than the original version). After large performance evaluation studies we found that $M D F=$ 10 provides the best tradeoff between energy consumption minimization and spectral efficiency. This is the value used in our simulation. It allows to make an adequate usage of the multiuser diversity in order to provide both, the same system capacity than MaxSNR and an energy minimization very close to the OEA results. However, considering a potential priority that could be required by operator in term of energy or system capacity, the value of $M D F$ could be always decrease or increase in order to meet the specific requirements.

\section{FETOT principle}

The FETOT scheduling principle is to allocate a TS of subcarrier $n$ to the user $k$ which provides the best "Bit Profitability" (" $B P_{k, n}$ ") in term of spectral efficiency, fairness and energy usage such as:

$$
B P_{k, n}=\frac{m_{k, n} * C F_{k}}{T E T_{k}}
$$

This dynamic priorities allows to significantly reduce energy consumption while optimizing the global system throughput and providing high fairness considering user location. Indeed, FETOT is designed to found, in the resource allocation, the user which provides the best trade-off between these three objectives : transmitting the maximum number of bit, consuming the less energy than possible and fairly share the RU across the time. This provides the profitable allocation in term of bit/Watt as well as good system capacity.

Thanks to the $B P_{k, n}$ parameters, higher priority are given to the users already awake but also to the users able to transmit the higher number of bit on the considered RU. Since $C n_{k}<<C_{k}+C n_{k}$, it is often more profitable in terms of energy consumption to continue to allocate the subcarriers to a same user rather to choose a new one for a negligible throughput gain. This allow to compress the user active mode session, maximizing the sleeping session duration and helping to reduce the energy consumption. However, if the active users experience poor radio condition, it will be more profitable to take benefit of the good potential throughput of a sleeping user which could experienced really better radio conditions due to low multipath fading. In this case, FETOT scheduler set this user in active mode and allocate to him the considered RU since it can provide a better ratio of transmitted bits by Watt, i.e a better bit transmission profitability. Contrary to OEA that give up a large part of the multiuser diversity usage benefit to decrease energy consumption, FETOT also make a more adequate usage of it thanks to the introduction of $M D F$ parameter. This parameter allows FETOT to reach better energy-throughput tradeoff activating an optimized number of users. Consequently FETOT highly improves the spectral efficiency compared to OEA with a negligeable supplementary energy cost. In addition, the FETOT resource allocation is done with fairness thanks to the $C F_{k}$ parameter that allows to have a same QoS for all user whatever their position from the access point.

\section{PERFormanCE EVAluations}

\section{A. Context and simulation setup}

Performance evaluation results are obtained using discrete event simulations that take into account path loss, shadowing and multi-path fading. In the simulations, we assume a total number $n_{s u b}$ of 32 subcarriers and a total number $n_{t s}$ of 50 TS in a frame. In addition, $C_{k}$ and $C n_{k}$ are fixed respectively equal to $110.2 \mathrm{~mW}$ and $46.8 \mathrm{~mW}$, for all $k$ in accordance with measured hardware consumption. The BER target is taken equal to $10^{-3}$. The Variable Bit Rate (VBR) source has a mean of $560 \mathrm{Kbps}$ and follows a poisson distribution. In order to study the influence of the distance on the scheduling performances, a first half of mobiles is situated close to the access point and have a mean $m_{k, n}$ equal to 8 bits. The second half are more far of the access point such as their mean $m_{k, n}$ equal to 6 bits. All performance criteria are done studying the influence of the traffic load. This one varies adding users 2 by 2 (each time, 1 close user and 1 far user).

\section{B. Global radio resources managements}

Fig. 2 shows the behaviours of each scheduler in the resource allocation. Fig. 2(a) represents the average number of TS used by each user in each frame. A TS is considered as "used" by a user if this last receive at least one RU of this TS in a frame and consequently that this user can not be set in sleep mode during this TS. The higher this value is, the more will be users activity duration (greedy in energy) and the more will be the energy consumption. Fig. 2(b) represents the mean number of allocated subcarrier to a same user per allocated TS. Fig. 2(c) shows the average global amount of RUs allocated to each user in a frame.

First, we can observe that with each scheduler, if we have only 2 user in the system, these users are not in hard competition with other since the system is highly underloaded. They receive all the subcarrier that they require to be satisfied on the first TSs of the frame (Fig. 2(b)). In average, after the allocation of all the subcarrier of approximately 3 TS (Fig. 2(a)), the users throughput requirements are satisfied and no more allocation has been done. Note that for only 2 users in the sytem, each scheduler provides very close allocation results since opportunistic scheduling show few benefits when multiuser diversity is very low.

Moreover, we can observe that, when the number of user increase, RR share the subcarrier of each TS with fairness between all users. This is due to the nature of the RR scheduling which alternativly serve user in RUs. Having less subcarrier per TS, each user need to use more TS which will induce more energy consumption. If we increase the number of user more than 24 (Fig. 2(a)), the system capacity is widely 


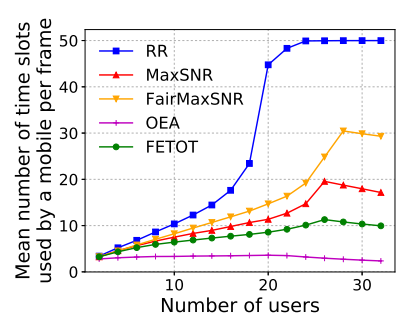

(a) TS allocations per frame.

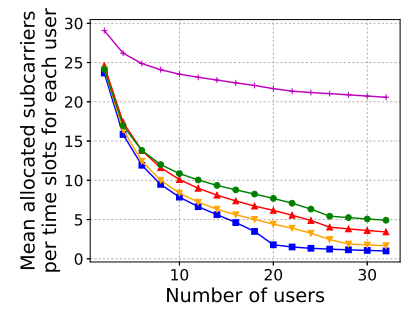

(b) Subcarriers allocations per TS.

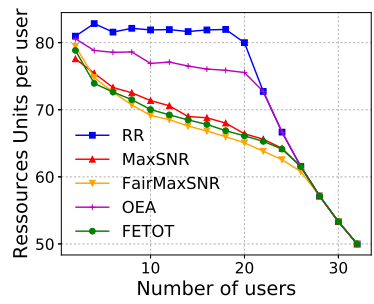

(c) Global RUs allocations.

Fig. 2. Schedulers radio resources managements.

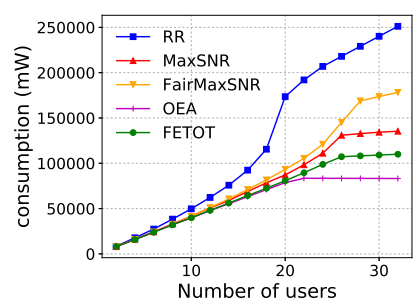

(a) Global system energy consumption.

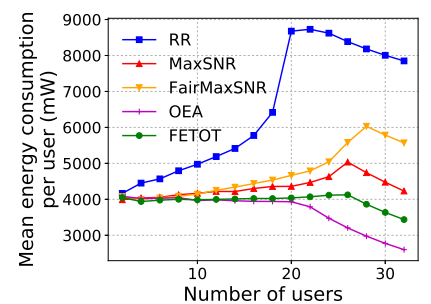

(b) Average energy consumed per user.

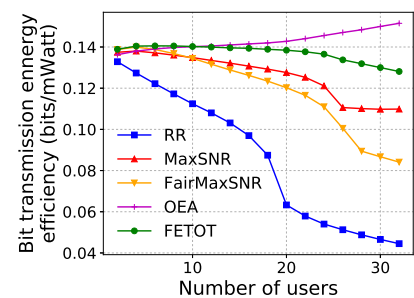

(c) Bit transmission energy efficiency.

Fig. 3. Impact of the scheduling strategies on the network energy consumption.

exceeded and each user consumes the maximum available TS in the system $\left(t_{\max }=50\right)$.

Regarding MaxSNR and FmaxSNR results, we can note that, like with RR, the subcarriers of each TS are shared between users (Fig. 2(b)). The higher the number of user is, the less is the number of subcarrier allocated to a user in a TS and the more is the number of TS needed by the users. However few differences exist with RR results. First, MaxSNR and FMaxSNR curves are above RR (Fig. 2(b)). In addition, the gradient of the curve is lower than RR curve (Fig.2(a)). Indeed, with these schedulers, the RUs are not simply shared between users but opportunistically allocated to the users with the best radio conditions during a frame. Consequently, it is statistically possible to have a same user with good condition on many subcarrier in a same frame. In addition, results of Fig. 2(c) show that MaxSNR and FMaxSNR take advantage of the multiuser diversity to maximise the number of bit transmitted per RU. Higher the number of users in the system is, the more efficient is the allocation process in term of system throughput since less RU are need by user (Fig. 2(c)). FMaxSNR allocates less subcarrier per TS to user since it is more fair than MaxSNR in the allocation process. indeed, it considers far user more regularly in the allocation process while close mobiles still have data to transmit (Fig. 2(b)). This explain why FmaxSNR allocates more TS to user per frame (i.e. less energy efficient) but need less RU to satisfy user than MaxSNR (better spectral efficiency due to a better multiuser diversity usage).

OEA encourages vertical resource mapping at the expense of multiuser diversity usage. This explains why the OEA curve is below the others in fig 2(a) and above in Fig.2(b). This shows that very few user are simultaneously in active mode. OEA wakes up only the minimum number of user in order keep acceptable spectral efficiency. Consequenlty, the TS's subcarriers are shared between users but only if necessary for the transmission energy profitability. This will ensure a strong trade-off between throughput and energy consumption in favour of the energy.

FETOT is an hybrid scheduler that is built on MaxSNR, FMaxSNR and Modified OEA solutions in order to combine their abilities. It tries to manage the most vertical resource mapping as possible while keeping a maximal benefit of the multiuser diversity. The result is a resource allocation thrifty in energy that satisfies users with less TS than with RR, MaxSNR and FMaxSNR 2(a) while offering good spectral efficiency by keeping a very low number of RU required to satisfy each user (Fig. 2(c)).

\section{Energy consumption}

Fig. 3(a) and Fig. 3(b) respectively show the global system and the user energy consumption. Fig. 3(c) shows the mean quantity of bit per $\mathrm{mW}$ that can be provided per each scheduler. Focusing on RR curves, we can observe that, between 2 and 20 users, the mean user's energy consumption increases with the number of users (Fig. 3(b)). This is due to the sharing of the TS's subcarrier between users (Fig.2(b)) which induces user activity duration increase (Fig.2(a)). Consequently, higher the number of user is, the more the subcarriers of each TS are shared between them and the more each user need TS. This induces that more the RR manage a large number of user, worst is its energy performances Fig. 3(a) and 3(c) ${ }^{1}$.

\footnotetext{
${ }^{1}$ With more of $20 \sim 22$ users, the system is overloaded and RR fails to provide the sufficient amount of RUs required by each user. They are often forced to stay in sleep mode even with data to transmit due to the lack of RUs. More often in sleep mode, the users consumed less energy over the time. This explains why with more than 22 user, the RR curve decrease (Fig. 3(b)).
} 


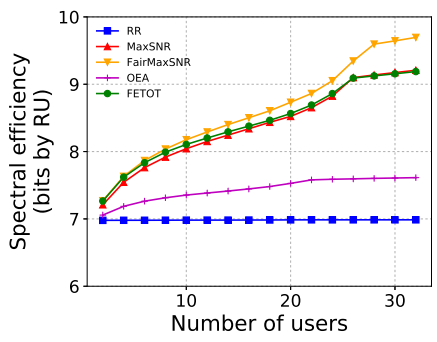

(a) Spectral efficiency.

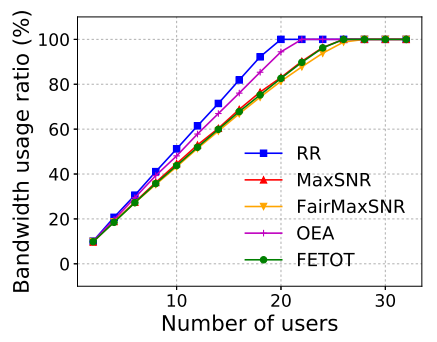

(b) Percentage of RU used.

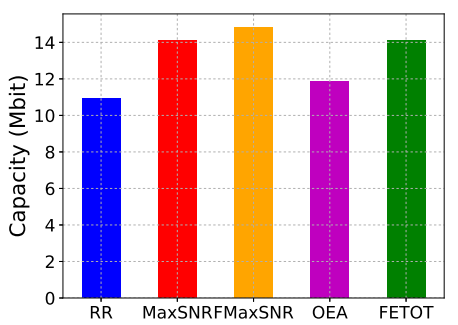

(c) Schedulers system capacity threshold.

Fig. 4. System capacity and spectral efficiency study.

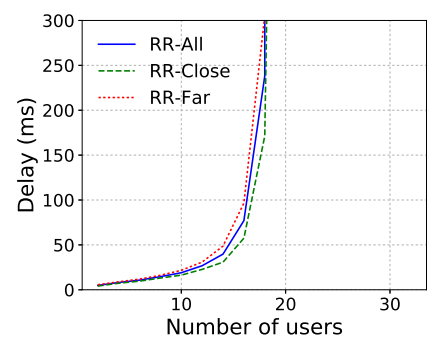

(a) Mean delay with RR algorithm.

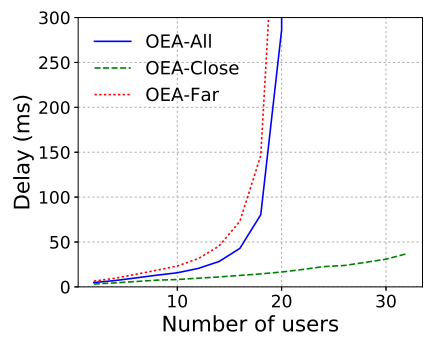

(d) Mean delay with OEA algorithm.

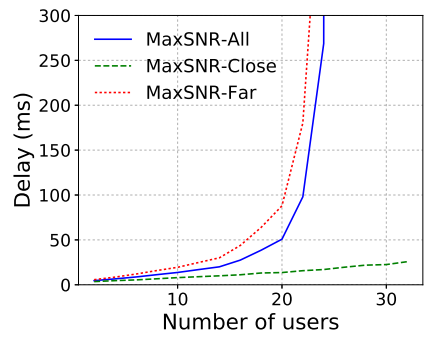

(b) Mean delay with MaxSNR algorithm.

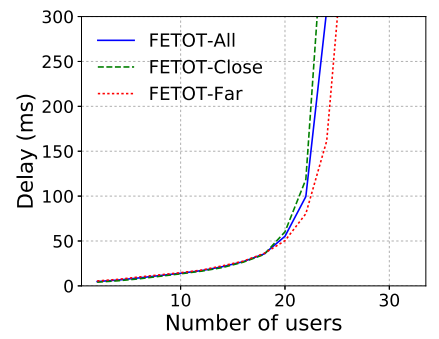

(e) Mean delay with FETOT algorithm.

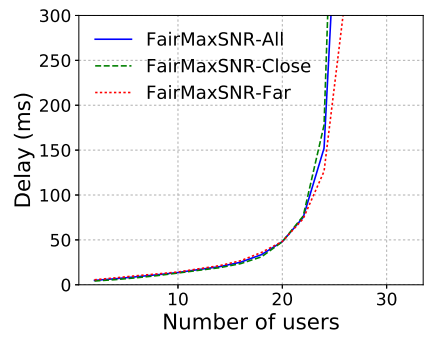

(c) Mean delay with FMaxSNR algorithm.

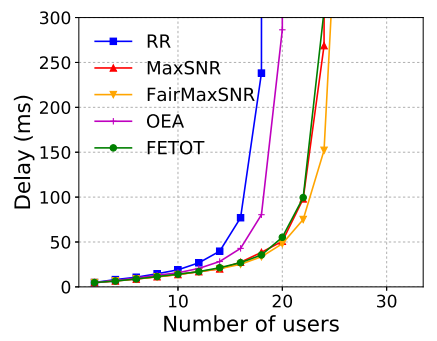

(f) Comparison of the average user delay obtain with each scheduler.

Fig. 5. Schedulers fairness regarding the delay of users at different positions.

Like RR, MaxSNR and FMaxSNR share the TS's subcarriers between users which provides an energy consumption increase with the traffic load Fig. 3(a). However this share is less fair at short time scale since it is statistically possible to have a same user with good condition on many subcarriers in a same frame. This corresponds to a more vertical allocation than RR. In addition, the usage of multi-user diversity allows to use less RUs by user when their number increase. This allows to reduce the user active mode duration and consequently the energy consumption. This explain why opportunistic scheduler curves grow more slowly than RR (Fig. 3(b) and 3(b)). Each Curve figure 3(b) decrease when the considered scheduler reach its congestion threshold/system capacity since after these traffic load threshold a part of users are forced to stay in sleep mode. Note that FMaxSNR use more energy than MaxSNR because it makes a more intensive usage of the multiuser diversity since he manage close and far mobile more fairly.

About OEA, we can observe that, instead to use all the multiuser diversity in order to exclusively improve system capacity, OEA preferes to sacrify a large amount of these benefits in order to also reduce energy consumption. Whatever the number of users in the system, the allocation process is thrifty. In addition, when the system capacity is exceeded with OEA (more than 20 22 users), the global energy consumption is stabilized to a low level. Contrary to other schedulers which wake up many users in order to transmit few bits simutanously on many TS's subcarriers, OEA maximizes the RUs utility (i.e. the number of bits transmitted per Watt consumed). Whatever the traffic load considered, few number of users are simultaneously activate in order to reach acceptable, but not energy expensive, spectral efficiency. Increasing the sleep mode duration, OEA provides an important energy gain if compared to other schedulers.

As expected, FETOT provides a tradeoff between OEA, FMaxSNR and MaxSNR energy results. Thanks to the introduction of the $M D F$ parameter, it succeed to make a better usage of the multiuser diversity with low energy supplementary cost Fig. 3(a)-3(c). With FETOT the number of bit that can be transmit per $\mathrm{mW}$ is higher than MaxSNR, FmaxSNR and RR and relativly close to OEA. Moreover, contrary than 
OEA that provides moderate spectral efficiency optimization, FETOT improve classical opportunistic energy consumption without negative side effect on system capacity.

\section{Spectral efficiency}

Fig. 4 shows the spectral efficiency of each allocation and their abilities to optimized or not the system capacity. RR scheduler does not take into account the radio condition and consequently does not take benefit of the multiuser diversity. Whatever the number of user, RR provides the same and poor spectral efficiency (Fig. 4(a)). At the opposite, MaxSNR is often acknowledged as the best scheduler regarding this network performance criterion. Indeed, at short time scale, allocating the RU at the user that can transmit the more appear as the most profitable. However at long time scale, it appears that the selected users are often close users of the access point. Since in realistic case, users have not always full buffer, sooner or later they will receive/transmit all their data and the MaxSNR will stay with only far users to manage. With half of the multiuser diversity, MaxSNR will not be able to always make the best bit/RU allocations. On the contrary, FMaxSNR makes a better usage of multiuser diversity thanks to more fair resource allocation. FMaxSNR considers all users, whatever their respective position, in a same step. Conserving more multiuser diversity accross the time, FairmaxSNR outperforms MaxSNR about spectral efficiency at long time scale (Fig. 4(a)). Consequently FMaxSNR also reachs its traffic congestion threshold latter than MaxSNR (noticable Fig. 4(c) and Fig. 4(b) where system overload is reach at $100 \%$ of the consumed RU). The OEA, which besides minimizes energy consumption, can not ensure the same level of throughput optimization. However, taking into account the radio condition variation in the allocation process, it stays partially opportunistic and provides a throuhput gain which is nevertheless significant compared to RR scheduler. FETOT is built and calibrated in order to take a maximal benefit of the multiuser benefit but while simulatneously avoiding to solicit user's wake up just for negligeable throughput gain. In addition FETOT integrates FMaxSNR fairness abilities that allows a good long term spectral efficiency. The result is that, despite FETOT provides high energy consumption minimization, it succeed to provide a same throughput efficiency than MaxSNR scheduler reaching the congestion to the same traffic load (Fig. 4(c) and Fig. 4(b)).

\section{E. Delay and fairness guarantee}

Fig. 5 shows the delay obtain with each schedulers in average for all users, in avarge for close usersand in average for far users. These results show that schedulers can be classified in 2 groups. RR, FmaxSNR and FETOT are fair guaranteeing slighly the same delay whatever the position of the user. MaxSNR and OEA are at the opposite, highly unfair. They always give the priority to the user with the most favorable ratio of bit per RU (for the maxSNR) or with the most favorable ratio of bit per watt than is equivelent to give the priority of closest user of the access point. The result is that they widely satisfied close user delay requirements even with high traffic load at the expense of the more far users that overpass their QoS requirement very quickly. Regarding and comparing the average delay of each scheduler computing all users delay whatever their distance from access point (Fig. 5(f)), RR provides the worst results due to a poor system capacity following by OEA that partially take benefit of the multiuser benefit. MaxSNR, FMaxSNR and FETOT provide the best results which must be analyzed without forget that FmaxSNR and FETOT are highly more fair than MaxSNR and that FETOT outperforms widely FmaxSNR is term of energy efficiency.

\section{CONClusion}

In the litterature many research effort have been done focusing on specific objectives : improve the system capacity (like MaxSNR), guarantee high fairness (like FMaxSNR) or minimize energy consumption (like OEA). However, if the proposed and acknowledged solutions are efficient on their specific issue, they often fail to meet the other performance criteria. This paper proposed a new approach that combines the advantages of each scheduler in order to provide the best tradeoff between global system throughput, fairness and energy efficiency. Futur works will focus to improve our solution studying the advantages to introduce a dynamic parameter, instead of $M D F$, in our algorithm based on the current traffic load.

\section{REFERENCES}

[1] C. Y. Wong and R. S. Cheng, "Multiuser OFDM with adaptive subcarrier, bit, and power allocation," IEEE J. Sel. Areas Commun., 1999.

[2] X. Wang and W. Xiang, "An OFDM-TDMA/SA MAC protocol with QoS constraints for broadband wireless LANs," ACM/Springer Wireless Networks, vol. 12, no. 2, pp. 159 - 170, 2006.

[3] P. Viswanath, D. N. C. Tse, and R. Laroia, "Opportunistic beamforming using dumb antennas," IEEE Transactions on Information Theory, vol. 48, pp. 1277 - 1294, June 2002.

[4] H. Kim, K. Kim, Y. Han, and J. Lee, "An efficient scheduling algorithm for QoS in wireless packet data transmission," in Proc. IEEE Int. Symposium on Personal, Indoor and Mobile Radio Communications (PIMRC), vol. 5, pp. 2244 - 2248, Sept. 2002.

[5] H. Kim, K. Kim, Y. Han, and S. Yun, "A proportional fair scheduling for multicarrier transmission systems," in Proc. IEEE Int. Vehicular Technology Conference (VTC), vol. 2, pp. 409 - 413, Sept 2004.

[6] C. Gueguen and S. Baey, "A fair opportunistic access scheme for multiuser OFDM wireless networks," Journal on Wireless Communications and Networking. European Association for Signal Processing (EURASIP). Special issue: Fairness in Radio Resource Management for Wireless Network, Feb. 2009.

[7] C. Gueguen and S. Baey, "A fair MaxSNR scheduling scheme for multiuser OFDM wireless systems," in Proc. IEEE Int. Symposium on Personal, Indoor and Mobile Radio Communications (PIMRC), 2009.

[8] M. R. del Olmo, R. Torrea-Duran, A. G. Orozco-Lugo, and M. Moonen, "Energy-efficient user scheduling algorithm for lte networks," in Proc. 5th Joint WIC/IEEE SP Symp. Inf. Theory Signal Process. Benelux (WIC), pp. 1-8, 2015.

[9] C. Gueguen, "Opportunistic energy aware scheduler for wireless networks," in Vehicular Technology Conference (VTC Spring), 2013 IEEE 77th, pp. 1-5, IEEE, 2013.

[10] V. de Beek et al., "A time and frequency synchronization scheme for multiuser OFDM," IEEE J. Sel. Areas Commun., vol. 17, Nov. 1999.

[11] S. K. Jayaweera, "Energy analysis of mimo techniques in wireless sensor networks," 38th Annual Conference on Information Sciences and Systems CISS 04, 2004.

[12] M. Andrews, K. Kumaran, and K. Ramanan, "Providing quality of service over a shared wireless link," IEEE Communications Magazine, vol. 39, pp. 150-154, Feb. 2001. 